\title{
Scaling Exponents for Barkhausen Avalanches in Polycrystalline and Amorphous Ferromagnets
}

\author{
Gianfranco Durin ${ }^{1}$ and Stefano Zapperi ${ }^{2}$ \\ ${ }^{1}$ Istituto Elettrotecnico Nazionale Galileo Ferraris and INFM, Corso M. d'Azeglio 42, I-10125 Torino, Italy \\ ${ }^{2}$ INFM sezione di Roma 1, Dipartimento di Fisica, Università "La Sapienza," P. le A. Moro 2, 00185 Roma, Italy
} (Received 7 July 1999)

\begin{abstract}
We investigate the scaling properties of the Barkhausen effect by recording the noise in several soft ferromagnetic materials: polycrystals with different grain sizes and amorphous alloys. We measure the Barkhausen avalanche distributions and determine the scaling exponents. In the limit of vanishing external field rate, we can group the samples in two distinct classes, characterized by exponents $\tau=$ $1.50 \pm 0.05$ or $\tau=1.27 \pm 0.03$, for the avalanche size distributions. We interpret these results in terms of the depinning transition of domain walls and obtain an expression relating the cutoff of the distributions to the demagnetizing factor which is in quantitative agreement with experiments.
\end{abstract}

PACS numbers: 75.60.Ej, 64.60.Ht, 68.35.Ct, 75.60.Ch

The Barkhausen noise is an indirect measure of complex microscopic magnetization processes and is commonly employed as a tool to investigate ferromagnetic materials [1]. In recent years, the interest for this phenomenon has grown considerably due to the connections with disordered systems and nonequilibrium critical phenomena.

Experiments have shown that the histogram of Barkhausen jump (avalanche) sizes follows a power law distribution [2-6], suggesting the presence of an underlying critical point $[4,7,8]$. This hypothesis implies that the statistical properties of the noise should be described by universal scaling laws, with critical exponents that are independent of the material microstructure, at least for some class of materials. However, the exact nature of the critical behavior is still debated. Several authors $[4,6,8,9]$ have analyzed the dynamics of flexible domain walls in random media relating the Barkhausen exponents to the scaling expected at the depinning transition. Other theoretical explanations involve a critical point tuned by the disorder in the framework of disordered spin models $[7,10]$.

The experiments reported in the literature can hardly resolve the theoretical issues since the measured exponents span a relatively wide range [2-5,11] and it is difficult to confirm whether universality holds. In particular, there has been no extensive and systematic measurement of critical exponents in different materials with homogeneous and controlled experimental conditions and reliable statistics. For example, experimental evidence of universality has recently been reported for acoustic emission avalanches recorded during martensitic transformations, by analyzing in detail the behavior of several alloys with different compositions [12].

The precise dependence of the Barkhausen characteristic sizes on experimentally tunable parameters is still a debated question $[4,6-9,11]$ and a complete agreement between theory, simulations, and experiments is still lacking. Understanding this point is crucial in order to link the material microstructure to the noise properties, or conversely to use the noise to obtain information on the structure of the material. In the past, this problem, which has important technological relevance, has been mainly addressed using phenomenological models $[1,13]$ where the material properties (i.e., grain size [14] and internal stresses [15]) are accounted for by effective fitting parameters. The main limit of this approach is that there is no systematic way to implement the program, without a precise understanding of the microscopic dynamics.

In this Letter, we report experimental data for a large set of materials suggesting the existence of two distinct universality classes and show that the results are in quantitative agreement with the theory of domain wall depinning transition. We perform Barkhausen noise measurements in six different ferromagnetic materials under similar experimental conditions, averaging the distributions over a large number $\left(\sim 10^{5}-10^{6}\right)$ of events, carefully testing the effect of the magnetic field rate on the exponents. The cutoff of the distributions is tuned by the demagnetizing factor and defines two new critical exponents, which we measure for two materials belonging to the different classes. Using scaling arguments, we predict values for these exponents that are in good agreement with experiments.

We record the Barkhausen noise using standard inductive methods, described in detail in Refs. [3,5,13]. A long solenoid provides a homogeneous low frequency triangular driving field, while a secondary pickup around the sample cross section gets the induced flux. The solenoid is $60 \mathrm{~cm}$ long, with a value of 1450 turns/meter, ensuring a homogeneous field up to $55 \mathrm{~cm}$ long samples with a peak amplitude of about $150 \mathrm{~A} / \mathrm{m}$. The pickup is made of 50 isolated copper turns, wound within $1 \mathrm{~mm}$. Such a small width is required to avoid spurious effects due to demagnetizing fields. All the measurements are performed only in the central part of the hysteresis loop around the coercive field, where domain wall motion is the relevant magnetization mechanism [1]. We take special care to reduce excess external noise during the measurements of avalanche distributions, as the evaluation of critical exponents is strongly affected by spurious noise. In this respect, the most appropriate cutoff frequency of the low pass preamplifier filter 
is chosen in the $3-20 \mathrm{kHz}$ range, roughly half of the sampling frequency, as usual in noise measurements.

The analysis of Barkhausen avalanche distribution is performed following the procedure discussed in Ref. [3]. We impose a reference level for $v_{r}$ for the signal $v(t)$, chosen above the background noise. The duration $T$ of the Barkhausen avalanches is defined as the interval within two successive intersections of the signal with the $v=v_{r}$ line. The avalanche size $s$ is calculated as the integral of the signal between the same points. We observe that the avalanche distributions follow a power law

$$
P(s)=s^{-\tau} f\left(s / s_{0}\right), \quad P(T)=T^{-\alpha} g\left(T / T_{0}\right),
$$

where $s_{0}$ and $T_{0}$ indicate the position of the cutoff to the power law behavior. The critical exponents result in being independent of the reference level for a reasonable range of $v_{r}$ [3].

We employ several different soft magnetic materials, both polycrystalline and amorphous: an $\mathrm{Fe}-\mathrm{Si} 7.8 \mathrm{wt} \%$ strip $(30 \mathrm{~cm} \times 0.5 \mathrm{~cm} \times 60 \mu \mathrm{m})$ produced by plan flow casting, annealed several times around $950{ }^{\circ} \mathrm{C}$ to obtain grains of average dimension of $25 \mu \mathrm{m}$; two strips of $\mathrm{Fe}-\mathrm{Si} \quad 6.5 \mathrm{wt} \%(30 \mathrm{~cm} \times 0.5 \mathrm{~cm} \times 45 \mu \mathrm{m})$, one annealed for $2 \mathrm{~h}$ at $1200{ }^{\circ} \mathrm{C}$, with grains of $160 \mu \mathrm{m}$, and the other annealed for $2 \mathrm{~h}$ at $1050{ }^{\circ} \mathrm{C}$, with grains of $35 \mu \mathrm{m}$ [16]. The amorphous samples have composition of the type $\mathrm{Fe}_{x} \mathrm{Co}_{85-x} \mathrm{~B}_{15}$ and we employ $\mathrm{Fe}_{21} \mathrm{Co}_{64} \mathrm{~B}_{15}$ as cast $(20 \mathrm{~cm} \times 1 \mathrm{~cm} \times 22 \mu \mathrm{m}), \mathrm{Fe}_{64} \mathrm{Co}_{21} \mathrm{~B}_{15}$ as cast $(28 \mathrm{~cm} \times 1 \mathrm{~cm} \times 23 \mu \mathrm{m})$. With these highly magnetostrictive alloys $\left[\lambda_{s} \sim(30-50) \times 10^{-6}\right]$ a tensile stress of $\sigma \sim 100 \mathrm{MPa}$ is applied during the measurement. The applied stress is found to enhance the signal-noise ratio, reducing biases in the distributions, but does not change the exponents [17]. A partially crystallized $\mathrm{Fe}_{64} \mathrm{Co}_{21} \mathrm{~B}_{15}$ $(22 \mathrm{~cm} \times 1 \mathrm{~cm} \times 23 \mu \mathrm{m})$ is also employed, with annealing for $30 \mathrm{~min}$ at $350{ }^{\circ} \mathrm{C}$ and then for $4 \mathrm{~h}$ at $300{ }^{\circ} \mathrm{C}$ under an applied tensile stress of $500 \mathrm{MPa}$. This induces the formation of $\alpha$-Fe crystals of about $50 \mathrm{~nm}$, with a crystal fraction of $\sim 5 \%$ [18].

In Fig. 1a we show the avalanche size distribution, obtained for the smallest available magnetic field rates ( $f=$ $3-5 \mathrm{mHz}$ ). We clearly see that the data can be grouped in two universality classes with $\tau=1.50 \pm 0.05$ and $\tau=$ $1.27 \pm 0.03$. The first class includes all the Si-Fe polycrystals and the partially crystallized amorphous alloy, while the amorphous alloys under stress belong to the second class. For the materials in the first class, we observed a linear decrease of the exponents on the frequency $f$ of the external magnetic field, in agreement with earlier findings [3]. The material in the second class does not show any noticeable dependence of the exponents on the field rate. We note that $\tau \simeq 1.3$, independent of the frequency, was previously measured in Perminvar [4]. Next, we measure the distribution of avalanche durations (see Fig. 1b) and find $\alpha=2.0 \pm 0.2$ and $\alpha=1.5 \pm 0.1$ for the two classes. The relatively large error bar should be considered
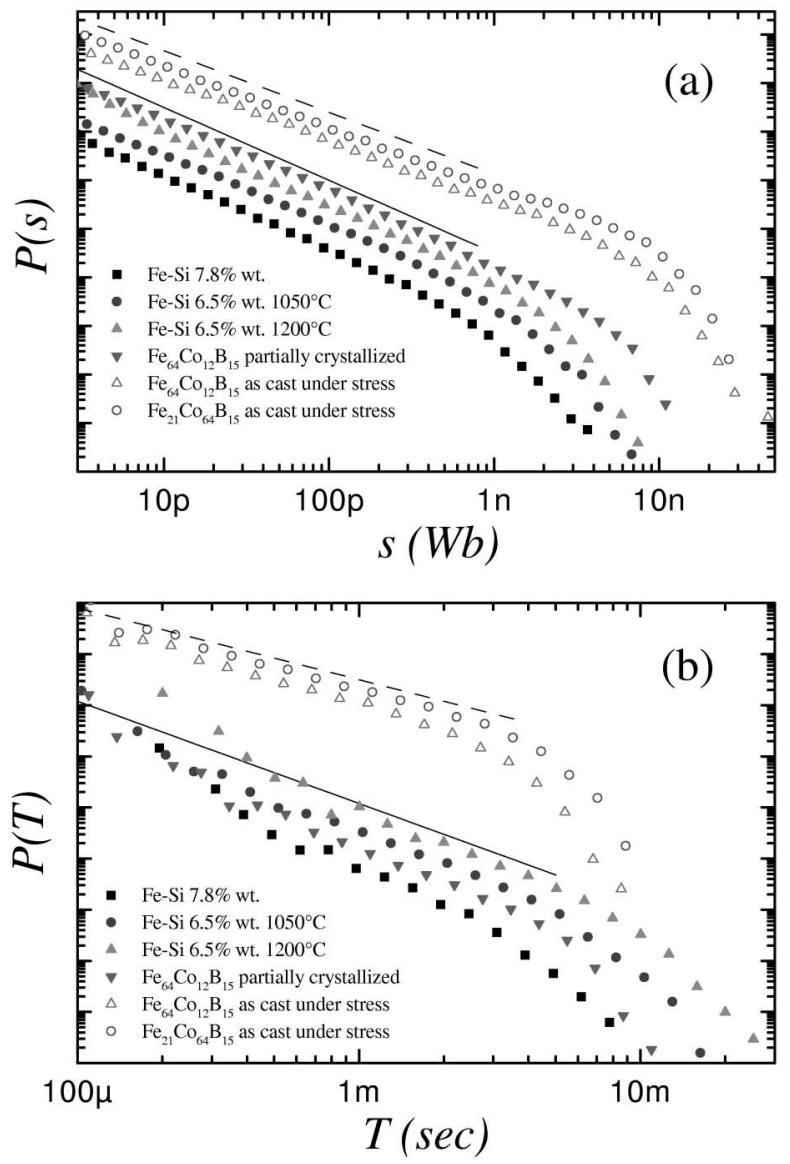

FIG. 1. (a) Distributions of Barkhausen jump sizes measured in different materials for the lowest available driving frequency. The solid line has a slope $\tau=1.5$ while for the dashed one $\tau=$ 1.27 , corresponding to the two universality classes. (b) Similar plot for duration distributions. The solid line has a slope $\alpha=2$, while for the dashed one $\alpha=1.5$.

as an upper limit and is due to the limited range of scaling and the presence of unavoidable excess external noise at low durations. Also in this case, $\alpha$ decreases linearly with $f$ for the materials belonging to the first class.

The scaling of the cutoff of Barkhausen avalanche distributions has been the object of an intense debate in the literature [6-9,11]. In Ref. [8] the control parameter was identified with the demagnetizing factor $k$. We thus measure the Barkhausen avalanche distributions varying $k$, using samples of different aspect ratios. In particular, we use the same sample and cut it progressively in shorter pieces, recording the noise always in the same region, whose size is limited by the pickup coil width. In this way only $k$ is varied, while stress, internal disorder and system size are kept constant. The demagnetizing factor is estimated as $k=1 / \mu_{c}-1 / \mu_{i}$, where $\mu_{c}$ is the linear permeability around the coercive field and $\mu_{i}$ is the intrinsic permeability (i.e., in an infinite strip) estimated using a magnetic yoke [19].

We perform the measurements on the Fe-Si 6.5 wt \% $1200^{\circ} \mathrm{C}$ (with lengths spanning from 28 to $10 \mathrm{~cm}$ ) and the $\mathrm{Fe}_{21} \mathrm{Co}_{64} \mathrm{~B}_{15}$ (from 27 to $8 \mathrm{~cm}$ ) under constant tensile 
stress. In Fig. 2 we report the avalanche size distribution for different $k$ for $\mathrm{Fe}_{21} \mathrm{Co}_{64} \mathrm{~B}_{15}$ in order to show the increase of the cutoff. Data collapse analysis yields $s_{0} \sim k^{-1 / \sigma_{k}}$ with $1 / \sigma_{k} \simeq 0.79$ (see Fig. 2 inset). Similarly the duration distribution cutoff scales as $s_{0} \sim k^{-\Delta_{k}}$ with $\Delta_{k} \simeq 0.4$. In the case of $\mathrm{Fe}-\mathrm{Si}$, the analysis is complicated by the frequency dependence of the exponents; therefore we fit the cutoff for different values of $f$ and extrapolate the results for $f \rightarrow 0$. The results for $s_{0}$ and $T_{0}$ for both materials are reported in Fig. 3 and Table I.

To interpret the experimental results we use the model of domain wall depinning discussed in Refs. [6,8]. A single $180^{\circ}$ domain wall is described by its position $h(\vec{r})$, dividing two regions of opposite magnetization directed along the $x$ axis. The total energy for a given configuration is the sum of different contributions due to magnetostatic, ferromagnetic, and magnetocrystalline interactions and gives rise to the following equation of motion $[6,8]$ :

$$
\begin{aligned}
\Gamma \frac{\partial h(\vec{r}, t)}{\partial t}= & 2 \mu_{0} M_{s} H-k \int d^{2} r^{\prime} h\left(\vec{r}^{\prime}, t\right) \\
& +\gamma_{w} \nabla^{2} h(\vec{r}, t)+\int d^{2} r^{\prime} J\left(\vec{r}-\vec{r}^{\prime}\right) \\
& \times\left[h\left(\vec{r}^{\prime}\right)-h(\vec{r})\right]+\eta(\vec{r}, h),
\end{aligned}
$$

where $\Gamma$ is the viscosity, $M_{s}$ is the saturation magnetization, $H$ is the applied field, $k$ is the demagnetizing factor, $\gamma_{w}$ is the surface tension of the wall, $J$ is the kernel due to dipolar interactions given by

$$
J\left(\vec{r}-\vec{r}^{\prime}\right)=\frac{\mu_{0} M_{s}^{2}}{2 \pi\left|\vec{r}-\vec{r}^{\prime}\right|^{3}}\left(1+\frac{3\left(x-x^{\prime}\right)^{2}}{\left|\vec{r}-\vec{r}^{\prime}\right|^{2}}\right),
$$

and $\eta(\vec{r}, h)$ is a Gaussian uncorrelated random field taking into account all the possible effects of dislocations, residual stress, and nonmagnetic inclusions.

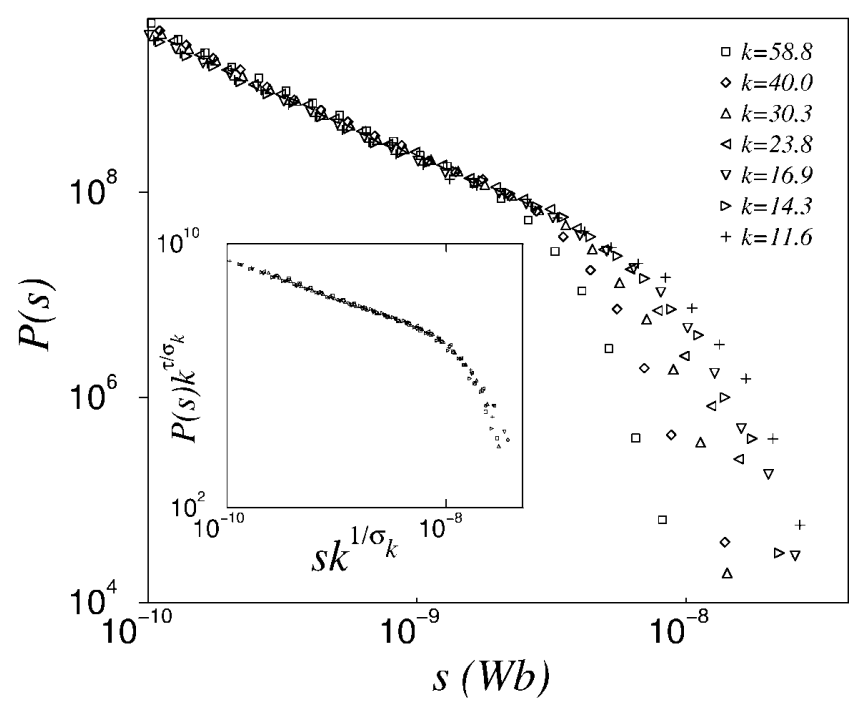

FIG. 2. The Barkhausen jump size distribution for different values of the demagnetizing factor $k$ in the $\mathrm{Fe}_{21} \mathrm{Co}_{64} \mathrm{~B}_{15}$ amorphous alloy under tensile stress The data collapse reported in the inset is done using $\tau=1.27$ and $1 / \sigma_{k}=0.79$.
The critical behavior of Eq. (2) has been understood using renormalization group methods [20-22], which show that at large length scales the critical exponents take meanfield values $[6,8]$. This result is due to the linear dependence on the momentum of the interaction kernel [Eq. (3)] in Fourier space [23]. In general, if we consider an interface whose interaction kernel in momentum space scales as $J(q)=J_{0}|q|^{\mu}$, the upper critical dimension is given by $d_{c}=2 \mu$, and the values of the exponents depend on $\mu$ (see Table I). In particular, Eq. (2) yields $\tau=3 / 2$ and $\alpha=2$ (i.e., $\mu=1$ ), or $\tau \simeq 1.27$ and $\alpha \simeq 1.5$ if dipolar interactions are neglected (i.e., $\mu=2$ ) [6]. Numerical simulations confirm the linear dependence of the exponents on the driving frequency $f$ for $\mu=1$ and no dependence for $\mu=2$ [17].

The experimental results are in perfect agreement with the values predicted using Eq. (2) and suggest that the dipolar interactions are stronger than surface tension effects in polycrystals, or whenever small grains are present, while in amorphous alloys under stress the surface tension is much stronger. Magnetostriction can be one of the sources of this effect, since the domain wall surface tension increases with stress $\sigma$ as $\gamma_{w} \sim \sqrt{K_{0}+3 / 2 \lambda_{s} \sigma}$, where $K_{0}$ is the zero applied stress anisotropy and $\lambda_{s}$ is the saturation magnetostriction [1,17]. Micromagnetic calculations for these particular materials are needed to have a final confirmation of this effect.

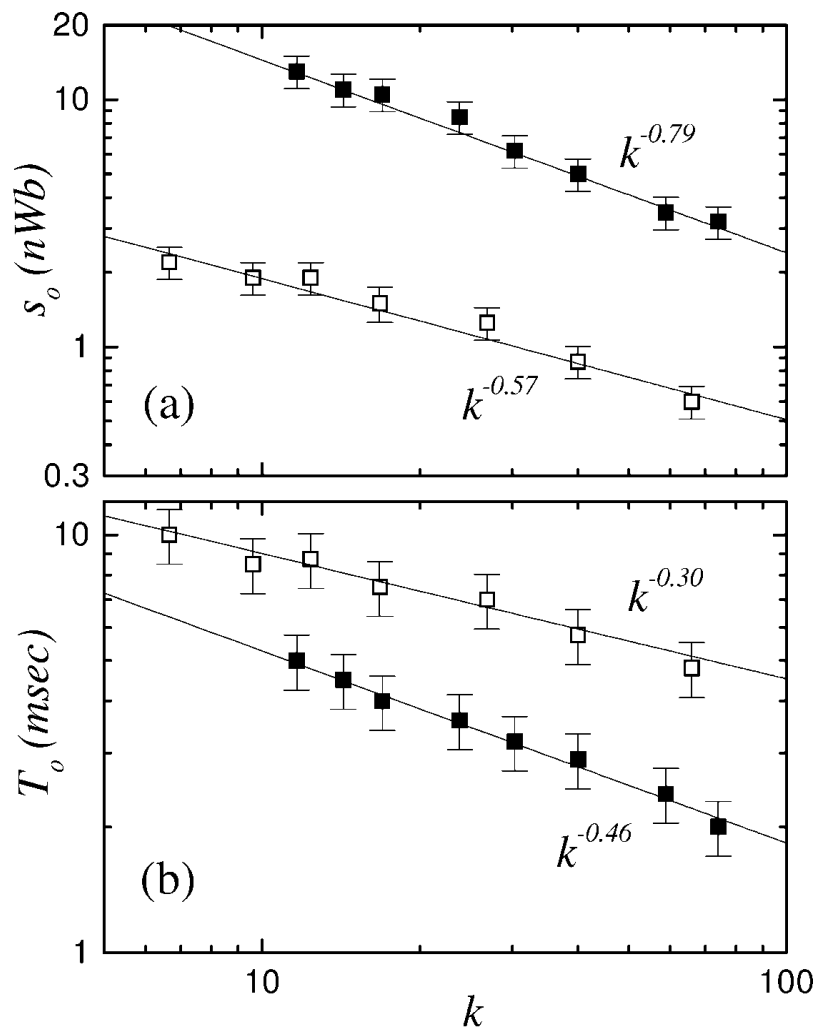

FIG. 3. The cutoff of the Barkhausen size (a) and duration (b) distributions as a function of $k$ in $\mathrm{Fe}-\mathrm{Si} 1200{ }^{\circ} \mathrm{C}$ (empty symbols) and $\mathrm{Fe}_{21} \mathrm{Co}_{64} \mathrm{~B}_{15}$ under constant tensile stress (filled symbols). 
TABLE I. The critical exponents measured in experiments compared with RG results [20-23] valid to order $\epsilon \equiv 2 \mu-d$ (see Ref. [6] for the scaling relations) and simulations for the two universality classes. System sizes range up to $L=150$ for $\mu=1$ and to $L=400$ for $\mu=2$.

\begin{tabular}{ccccc}
\hline \hline$\mu=1$ & $\tau$ & $\alpha$ & $1 / \sigma_{k}$ & $\Delta_{k}$ \\
RG & $3 / 2$ & 2 & $2 / 3$ & $1 / 3$ \\
Sim. & $1.50 \pm 0.02$ & $2.00 \pm 0.05$ & $0.65 \pm 0.05$ & $0.3 \pm 0.1$ \\
Expt. & $1.50 \pm 0.05$ & $2.0 \pm 0.2$ & $0.57 \pm 0.10$ & $0.30 \pm 0.10$ \\
$\mu=2$ & $\tau$ & $\alpha$ & $1 / \sigma_{k}$ & $\Delta_{k}$ \\
RG & $5 / 4$ & $10 / 7$ & $2 / 3$ & $7 / 18$ \\
Sim. & $1.27 \pm 0.02$ & $1.50 \pm 0.05$ & $0.72 \pm 0.03$ & $0.39 \pm 0.05$ \\
Expt. & $1.27 \pm 0.03$ & $1.5 \pm 0.1$ & $0.79 \pm 0.10$ & $0.46 \pm 0.10$ \\
\hline \hline
\end{tabular}

We derive the dependence of the cutoff on $k$ from Eq. (2) noting that the demagnetizing field acts as a restoring force for the interface motion and is responsible for the cutoff in the avalanche sizes. The interface cannot jump over distances larger than $\xi$, defined as the length for which the interaction term $J_{0}|q|^{\mu}$ is overcome by the restoring force (i.e., $J_{0} h \xi^{-\mu} \sim k \xi^{d} h$ ) which implies [24]

$$
\xi \sim\left(k / J_{0}\right)^{-\nu_{k}} \quad \nu_{k}=1 /(\mu+d) .
$$

The avalanche size and duration distribution cutoffs can be obtained using the scaling relations reported in Ref. [6]:

$$
s_{0} \sim D\left(k / J_{0}\right)^{-1 / \sigma_{k}}, \quad 1 / \sigma_{k}=\nu_{k}(d+\zeta)
$$

and similarly

$$
T_{0} \sim D\left(k / J_{0}\right)^{-\Delta_{k}}, \quad \Delta_{k}=z \nu_{k},
$$

where $D \equiv \sqrt{\left\langle\eta^{2}\right\rangle}$ denotes the typical fluctuation of the disorder. The dynamic exponent $z$ and the interface roughness exponent $\zeta$ define the spatiotemporal scaling of the domain wall width $\sqrt{\left\langle h^{2}\right\rangle-\langle h\rangle^{2}}=\xi^{\zeta} F\left(t / \xi^{z}\right)$.

Inserting in Eqs. (5) and (6) the renormalization group results [20-23] $\zeta=(2 \mu-d) / 3$ and $z=\mu-(4 \mu-$ $2 d) / 9$, we obtain $1 / \sigma_{k}=2 / 3$ and $\Delta_{k}=[\mu-(4 \mu-$ $2 d) / 9] /(\mu+d)$. We have performed extensive numerical simulations in $d=2$ using the model described in Ref. [6] in order to test these results (see Table I). Our results also agree with earlier numerical simulations in $d=1$ for $1<\mu<2$, where $s_{0} \sim k^{-0.65}$ independent of $\mu$ [25] and with the result reported in Ref. [11] (i.e., $s_{0} \sim L^{1.4}$ ) obtained using $k \equiv 1 / L^{2}$.

The scaling of the cutoff is different for a local demagnetizing field $-k h(x, t)$ [4], which yields $\nu_{k}=1 / \mu$ [9]. However, a nonlocal term is more appropriate to describe the demagnetizing field, due to the long range of magnetostatic interactions [6], as is confirmed by the agreement between experiments and theory.

In conclusion, our experiments suggest that the Barkhausen effect can be described by universal scaling functions and that materials can be classified in different universality classes. The theory of interface depinning can be used to obtain a quantitative explanation of the experiments, providing a natural framework to understand the properties of soft magnetic materials.

[1] G. Bertotti, Hysteresis in Magnetism (Academic Press, San Diego, 1998).

[2] G. Bertotti, G. Durin, and A. Magni, J. Appl. Phys. 75, 5490 (1994).

[3] G. Durin, A. Magni, and G. Bertotti, Fractals 3, 351 (1995).

[4] J. S. Urbach, R. C. Madison, and J. T. Markert, Phys. Rev. Lett. 75, 276 (1995).

[5] D. Spasojevic, S. Bukvic, S. Milosevic, and H. E. Stanley, Phys. Rev. E 54, 2531 (1996).

[6] S. Zapperi, P. Cizeau, G. Durin, and H.E. Stanley, Phys. Rev. B 58, 6353 (1998).

[7] O. Perkovic, K. Dahmen, and J. P. Sethna, Phys. Rev. Lett. 75, 4528 (1995).

[8] P. Cizeau, S. Zapperi, G. Durin, and H.E. Stanley, Phys. Rev. Lett. 79, 4669 (1997).

[9] O. Narayan, Phys. Rev. Lett. 77, 3855 (1996).

[10] E. Vives, J. Goicoechea, J. Ortín, and A. Planes, Phys. Rev. E 52, R5 (1995).

[11] M. Bahiana et al., Phys. Rev. E 59, 3884 (1999).

[12] L. Carrillo, L. Mañosa, J. Ortín, A. Planes, and E. Vives, Phys. Rev. Lett. 81, 1889 (1998).

[13] B. Alessandro, C. Beatrice, G. Bertotti, and A. Montorsi, J. Appl. Phys. 68, 2901 (1990); 68, 2908 (1990).

[14] G. Bertotti and A. Montorsi, J. Magn. Magn. Mater. 86, 214 (1990).

[15] M. J. Sablik, J. Appl. Phys. 74, 5898 (1993).

[16] C. Appino et al., J. Phys. IV (France) 8, Pr2-531 (1998).

[17] G. Durin and S. Zapperi, J. Appl. Phys. 85, 5196 (1999).

[18] V. Basso et al., J. Magn. Magn. Mater. 157/158, 217 (1996).

[19] The error bar for $k$ is considerably smaller than the error bars for $s_{0}$ and $T_{0}$, although, in principle, an incorrect estimate of $\mu_{i}$ could give rise to a systematic error.

[20] T. Nattermann, S. Stepanow, L. H. Tang, and H. Leschhorn, J. Phys. II (France) 2, 1483 (1992).

[21] O. Narayan and D. S. Fisher, Phys. Rev. B 48, 7030 (1993).

[22] H. Leschhorn, T. Nattermann, S. Stepanow, and L. H. Tang, Ann. Phys. (Leipzig) 6, 1 (1997).

[23] D. Ertas and M. Kardar, Phys. Rev. E 49, R2532 (1994).

[24] Contrary to the other exponents, $\nu_{k}$ does not receive loop corrections $[9,21]$.

[25] A. Tanguy, M. Gounelle, and S. Roux, Phys. Rev. E 58, 1577 (1998). 